\title{
Germanica
}

\section{«Sujet impossible » suivi de «Ich will seicht sein» et d'un entretien avec Elfriede Jelinek}

«Das unmögliche Subjekt» mit einem Essay von Elfriede Jelinek («Ich will seicht sein») und einem Interview mitihr

\section{Yasmin Hoffmann}

\section{OpenEdition}

\section{Journals}

Édition électronique

URL : https://journals.openedition.org/germanica/2046

DOI : 10.4000/germanica.2046

ISSN : 2107-0784

Éditeur

Université de Lille

Édition imprimée

Date de publication : 30 juin 1996

Pagination : 153-175

ISBN : 9782098426320

ISSN : 0984-2632

\section{Référence électronique}

Yasmin Hoffmann, « «Sujet impossible » suivi de «lch will seicht sein» et d'un entretien avec Elfriede Jelinek », Germanica [En ligne], 18| 1996, mis en ligne le 14 juin 2013, consulté le 21 septembre 2021 URL : http://journals.openedition.org/germanica/2046 ; DOl : https://doi.org/10.4000/germanica.2046

Ce document a été généré automatiquement le 21 septembre 2021.

(c) Tous droits réservés 


\section{«Sujet impossible » suivi de «Ich will seicht sein» et d'un entretien avec Elfriede Jelinek}

«Das unmögliche Subjekt» mit einem Essay von Elfriede Jelinek («Ich will seicht sein») und einem Interview mitihr

\section{Yasmin Hoffmann}

In chinesischen Legenden steht geschrieben, daß große Meister in ihre Bilder hineingingen und verschwunden sind. Die Frau ist kein großer Meister. Deshalb wird ihr Verschwinden nie

vollkommen sein. Sie taucht wieder auf, beschäftigt wie sie ist, mit dem Verschwinden.

Eva Meyer

Cinq romans La Pianiste (1983), Les Exclus (1980), Lust (1989), Les Amantes (1975), Méfionsnous de la nature sauvage $(1985)^{1}$, deux pièces de théâtre Ce qui arriva quand Nora quitta son mari ${ }^{2}$, et Totenauberg ${ }^{3}$, ainsi que le scénario de Malina écrit pour le film de Werner Schröter, ont permis au public français de se faire une idée de la diversité de l'écriture d'Elfriede Jelinek, musicienne de formation, romancière, essayiste, auteur de nombreuses pièces radiophoniques, et femme de théâtre. Si sa réputation de dramaturge n'est plus à faire dans les pays germaniques, ses pièces ont mis néanmoins une quinzaine d'années avant de s'imposer sur les scènes autrichiennes et allemandes et se sont heurtées à des résistances importantes. Résistances à la mesure de la violence du propos tenu, car toute l'écriture d'Elfriede Jelinek est trangression et défi. Ses pièces ont la réputation d'être « injouables », ce qui signifie qu'elles sont un défi pour la scène. Lors d'une discussion après la première de Krankheit oder Moderne Frauen Heiner Müller formulait ce défi ainsi :

Was mich daran interessiert: Es gibt eine so grundsätzliche Schwierigkeit zwischen Drama und Theater, daß Stücke oder Texte, die dem Theater nachlaufen, sehr schnell auf sehr viele Bühnen kommen, und Texte, die dem Theater etwas 
voranlaufen, etwas schwer und ganz selten auf die Bühne kommen. Was mich interessiert an den Texten von Elfriede Jelinek, ist der Widerstand, den sie leisten gegen das Theater, so wie es ist ${ }^{4}$ pièces d'Elfriede Jelinek n'en demandent pas trop à la scène, si ses pièces ne sont pas écrites contre le théâtre, contre une certaine conception du théâtre :

Ihre Figuren sprechen keine Dialoge, alles Menschliche ist ihnen fremd; sie sind Sprechmaschinen, Sprachgesangautomaten. Mann-Deutsch kämpft gegen FrauDeutsch, Dumm-Deutsch gegen Blut-Deutsch. Die Männer verwandeln sich im Verlauf des Stücks von Sprechmaschinen in Sprachvernichtungsmaschinen [...] als seien [sie] Überlebende aus Karl Kraus' Letzten Tage der Menschheit, Flüchtlinge aus Ernst Jandls Lautgedichten ${ }^{5}$.

3 Elfriede Jelinek considère la scène comme un lieu totalement artificiel, le jeu des acteurs ne doit refléter aucune psychologie, et si son théâtre est difficile à mettre en scène c'est qu'il est avant tout discours. Discours sur l'inégalité des sexes e.a. pour Clara. S., Nora ${ }^{6}$ et Krankheit ${ }^{7}$. Dans Krankheit oder Moderne Frauen, et à travers la double invraisemblance des deux vampires féminins - héroïnes de la pièce -, sont mis en scène des mythes, des monstres, et par là-même des fantasmes et des peurs. "Je suis malade, donc je suis ", affirme l'une des femmes-vampires, se moquant du rationalisme d'une humanité émancipée. Et de quoi souffre-t-elle? D'une maladie sexuellement transmissible que serait la féminité ? En partie seulement, car la maladie qui envahit la scène ce seraient plutôt les clichés, les modèles culturels, les stéréotypes, les discours politiques et slogans médiatiques qui ont contribué et contribuent toujours à distribuer les rôles dans la société. Elfriede Jelinek s'attaque volontiers aux mythes, aux grands comme à ceux de la vie quotidienne. Satiriste et moraliste, elle l'est par bien des aspects, dans la lignée d'un Karl Kraus découvrant et faisant découvrir des "abîmes là où se nichent des lieux communs ». Ecrivain engagé, elle l'est aussi, et sa férocité est à la mesure de la monstruosité de l'idéologie véhiculée par des clichés, les stéréotypes, le proverbial, les modèles collectifs.

4 Mais si l'on voulait se faire une idée plus juste de l'esprit sous-jacent à son théâtre, il faudrait évoquer Artaud, rêvant d'un théâtre essentiel, d'un théâtre de la cruauté que le spectateur ressentirait comme une morsure, et qui serait comparable à la peste. Ce théâtre-là, d'après Artaud, ne serait pas contagieux, mais, «comme la peste, il serait révélation, la mise en avant, la poussée vers l'extérieur d'un fond de cruauté latente par lequel se localiseraient sur un individu ou sur un peuple toutes les possibilités perverses de l'esprit $»^{8}$. Le sang qui coule dans Krankheit n'est pas un sang innocent, ce n'est pas le sang d'un héros, ni le sang de la victime ou de la vengeance, c'est le sang de la femme, de la parturiente, comme il en coule rarement sur la scène, un sang des plus impurs, touchant au plus profond des tabous lorsqu'il coule en public. Il provoque des peurs face à un danger qui vient de l'intérieur de l'identité sexuelle et/ou sociale ; c'est un sang qui menace les rapports entre les sexes dans la société et qui, par l'intériorisation des identités sexuelles respectives, menace l'identité même du sexe, puisqu'il est le symbole par excellence de la différence sexuelle. Les représentations symboliques d'univers utérins sont courantes sur scène et dans la littérature, il suffit de penser aux nombreuses grottes et cavernes, aux représentations de la fertilité, etc. Plus rares en revanche sont les accouchements en direct sur scène. Et encore plus rares les femmes-vampires assises sur un fauteuil mi-gynécologique, mi-dentaire et que l'accoucheur vide comme on vide un poulet. La scène est difficile à surpasser en 
grotesque, et certains diront en matière de provocation et de mauvais goût, car pendant l'accouchement les hommes sortent de curieux objets de la femme: des ballons gonflables, des jouets en plastique, des canards, des grenouilles, des cygnes, etc. Voici que le mythe de l'éternel féminin, de l'éternelle tentatrice accouche de pacotilles, et il faut bien dire qu'Emily et Carmilla, les deux vampires, n'ont plus le mordant de leurs ancêtres: elles ont perdu la possibilité donnée aux mythes d'inciser des abcès collectifs qu'ils soient de nature sociale ou morale. La cruauté de la pièce ne réside pas tant dans la « monstruosité » de ces femmes qui tuent leurs enfants pour les congeler et les manger plus tard - la tragédie grecque abonde en atrocités comparables -, elle résiderait plutôt dans l'impossibilité tragicomique du vampire à devenir le sujet d'une tragédie, à accéder au statut d'une Médée, comme le fait remarquer le mari de l'une d'elle : «Carmilla, eines sage ich dir: Eine Medea wirst du trotzdem nicht! Du bist und bleibst eine Hausfrau. Wenn du nun stirbst, bist du eine tote Hausfrau.» (p. 54). L'indication scénique à elle seule permet de mesurer l'étendue de la déchéance des Médée :

Jetzt sieht die Bühne folgendermaßen aus: Die Arztpraxis ist verschwunden. Dafür ein reizendes Schlafzimmer im Stil der 50er Jahre, Ehebetten [...], Nachtkästchen, Lämpchen, ein Radio etc. Nur statt der Betten, stehen elegant gefertigte mit Erde gefüllte Särge im Stil dieser 50er da. Idyll. [...] Ab und zu flattert die uns schon bekannte Fledermaus darüber hin. Ab und zu flattert ein gemeiner Vogel herum und schreit. So ist es gut. Danke. Links und rechts in den Ehebetten liegen gemütlich Emily und Carmilla, letztere mit Lockenwicklern. Emily hat eine Reiseschreibmaschine auf einem Brett stehen, das über den Sarg gelegt ist. Gut sichtbar zwei Tiefkühltruhen: «Familie». (p. 41)

Autre femme, autre mythe: La Nora. Cent ans exactement séparent la Nora d'Ibsen (1879) de la Nora d'Elfriede Jelinek, dont la première représentation eut lieu en 1979. Cent ans d'histoire que l'on pourrait placer sous le signe de la perversion et qui trouverait sa forme accomplie dans la figure du couple sado-masochiste au centre de la pièce de Jelinek. Cent ans d'histoire d'adaptation sociale, basée sur l'intériorisation des contraintes et la soumission, sur le plaisir et l'oppression. Comme Ingeborg Bachmann, Elfriede

6 Jelinek traque la continuation du fascisme à l'intérieur de la cellule familiale et conjugale et comme Bachmann elle montre que «le massacre a lieu à l'intérieur de ce qui est permis, de la bienséance, à l'intérieur d'une société aux nerfs fragiles, qui frémit d'horreur devant les atrocités commises $»^{9}$. Pour cela elle agrandit ses personnages jusqu'à les rendre monstrueux :

«Je magnifie des choses en les écrivant, je les mets sur un piédestal. Tous mes personnages sont plus grands que nature. C'est pourquoi ils ne cadreraient pas dans des films, à moins qu'il ne fussent transposés par un Werner Schröter ou le regretté Fassbinder. Ces deux là étaient capables d'utiliser une langue artificielle dans un dialogue. ${ }^{10}$

7 Elfriede Jelinek a imaginé le destin de Nora après son départ de la maison de poupée. Elle a imaginé le trajet qui mène de l'objet (la petite poupée) au sujet, qui essaie de vivre par ses propres moyens en voulant se faire embaucher comme ouvrière dans une usine de textile. Mais ces moyens se révèlent être des moyens propres à tout un réseau de déterminations économiques et sociales :

NORA: Ich bin keine Frau, die von ihrem Mann verlassen wurde, sondern eine, die selbsttätig verließ, was seltener ist. Ich bin Nora aus dem gleichnamigen Stück von Ibsen. Im Augenblick flüchte ich aus einer verwirrten Gemütslage in einen Beruf. 
PERSONALCHEF: An meiner Position können Sie studieren, dass ein Beruf keine Flucht, sondern eine Lebensaufgabe ist.

NORA: Ich will aber mein Leben noch nicht aufgeben! Ich strebe meine persönliche Verwirklichung an. (p. 7)

8 Avec l'homme nous entrons dans le registre des lieux communs : vie bien remplie par le travail, métier, profession qui permet l'épanouissement de toute une vie; puisque le chef du personnel, lui, entend par «Lebensaufgabe»: «eine Aufgabe für das Leben». Nora ne l'entend pas tout à fait ainsi et à travers sa réponse matérialise l'autre aspect contenu dans la «Lebensaufgabe», celui de la mort et du renoncement, «die Aufgabe» devient sacrifice, vie sacrifiée, mise à mort. Et en effet le travail que le chef du personnel va proposer à Nora n'est pas un métier à proprement parler, mais une exécution idéologique de sa personnalité :

PERSONALCHEF: [...] Wir verfügen über Maschinen Vor einer Maschine muß der Mensch zu einem Nichts werden, erst dann kann er wieder zu einem Etwas werden. Ich allerdings wählte von Anfang an den beschwerlicheren Weg zu einer Karriere, (p. 7)

9 Voici que sur le versant masculin la «Lebensaufgabe» devient carrière, réalisation d'ambitions professionnelles, aux antipodes du devoir et du sacrifice qui incombent à la femme. À souligner aussi le «allerdings» qui sépare le chef du personnel du reste de la masse travailleuse. Si les ouvriers veulent devenir "quelque chose» il faut d'abord qu'ils soient réduits à néant, affirme-t-il, or ce qui attend les ouvriers et ouvrières va du "quelque chose» vers le "rien", vers l'anéantissement. L'usine de textile faisant l'objet de spéculations immobilières qu'il s'agit de cacher aux ouvriers. L'important est de savoir qui se sert de telles expressions et à quelle fin. Ici le contenu idéologique de la «Lebensaufgabe» est démasqué par la réplique de Nora qui fait dériver du substantif un verbe, certes contenu dans la polysémie préexistante, mais qui dans sa réponse signifie mourir. A travers ce jeu de mots ou glissement de sens il apparait clairement qu'il y a un monde entre la «Lebensaufgabe» d'un homme et celle d'une femme. Pour l'homme ce monde signifie carrière, pour la femme il signifie destin et - disparition.

Bien que cette pièce fut écrite à l'apogée du mouvement féministe, à une époque où les espoirs politiques étaient encore permis, la distance ironique et le pessimisme culturel inhérent à toute l'œuvre d'Elfriede Jelinek l'excluent d'emblée en tant que pièce militante où s'exprimerait l'euphorie du moment. En fait c'est un montage où s'entremêlent personnages et actions de deux pièces d'Ibsen: "Nora, la maison de poupée » (1879) et «Les soutiens de la société » (1878). Si Elfriede Jelinek situe sa pièce dans les années vingt, à l'époque du fascisme montant en Allemagne, à une époque où le langage du capital était moins voilé, plus cru, c'est pour mieux mettre en valeur un des aspects de la société libérale : le jeu froidement calculé d'intérêts. C'est aussi le temps des grandes spéculations et des faillites tout aussi spectaculaires.

11 Comme tous les personnages d'Elfriede Jelinek, Nora est néanmoins un personnage de composition au sens propre, elle tient autant de l'héroïne d'un roman à l'eau de rose que d'un modèle proposé par la presse féminine ou les mass média. Les ouvrières à l'usine lui renvoient le reflet de ce qu'elle pourrait, mais ne voudrait à aucun prix être. Ce n'est pas un personnage en mesure de rester à la hauteur de l'idéalisme et de l'héroïsme du personnage d'Ibsen. Et lorsque peu après son arrivée à l'usine le contremaître ainsi que le Consul Weygang, un grand spéculateur et industriel, s'éprennent d'elle, Nora n'hésite pas un instant : 
VORARBEITER: Du mußt bei mir bleiben, Nora! Du kannst nicht mit einem fremden Mann mitgehn.

NORA $z u$ Vorarbeiter: Ein Mann muß auch lernen zurückzustehen vor dem Stärkeren. Das ist in der Natur so weise eingerichtet.

VORARBEITER: Geh nicht, Nora! Ich werde dich sicher hier herausholen können! Ich werde unermüdlich Kurse besuchen und Aufstiegschancen wahrnehmen.

[...] NORA: Ich liebe ihn.

VORARBEITER: Du liebst nur sein Geld!

NORA: Schon einmal ist Geld ein schlimmer Abstieg für mich gewesen, ein zweites Mal werde ich einen Aufstieg nehmen. Geld will ich diesmal von meiner Liebe fernhalten (p. 21).

12 Scène après scène le spectateur assiste à la perte progressive et tragi-comique de toutes les illusions. L'espoir se réduit comme une peau de chagrin, l'espoir de l'objet de devenir sujet. La petite poupée a certes quitté son «destin naturel» de mère et d'épouse, mais elle reste un objet de convoitises, totalement attachée à une conception stéréotypée du bonheur, de la sécurité etc. D'où sa liaison avec Weygang auprès duquel elle apprendra bien vite que les charmes de la séduction ne sont pas éternels, que « les femmes sont une denrée hautement périssable ». Elle comprendra tout aussi vite qu'il lui faut devenir l'instrument d'intérêts économiques - ceux de son nouveau compagnon précisément qui veut se servir de ses charmes à des fins d'espionnage industriel jusqu'au rabaissement, à la dépravation totale: se déguiser en prostituée pour masochistes afin de recueillir des secrets économiques - auprès de son premier mari : Torvald Helmer !

Pour Elfriede Jelinek cette histoire est significative du rôle que les femmes ont joué dans l'idéologie fasciste. Consciemment Nora a pactisé avec Weygang au nom d'intérêts économiques, et ce faisant elle a signé son propre arrêt de mort. Cette deuxième mort Nora la vivra en retournant chez Helmer, lui aussi ruiné par Weygang. Et les voilà tous deux de retour à la case départ, la misère en plus. Tandis les instructions scéniques indiquent que la musique fasciste lentement envahit la scène, gageons que tous deux sauront sortir de leur misère en misant sur cette nouvelle valeur montante.

L'on chercherait en vain un discours positif dans la Nora d'Elfriede Jelinek. Le salut ne viendra ni de la lutte des classes, ni des femmes. Et l'espoir ne réside pas non plus dans une hypothétique redistribution des rôles dans la société :

WEYGANG: Nach all den vielen vielen Monaten kommt der Ernst des Lebens, [...] Könnte meine Heidelerche Verantwortung tragen? Mir ein echter Partner sein? Der Partnertyp ist eine Art Frau, die langsam modern zu werden beginnt.

NORA: Ich bin aber mehr eine altmodische Frau, die ganz hinter den Mann zurücktritt, daß man nur mehr ihn sieht. (p. 30)

Un personnage comme Nora, autrefois héroïne d'une éducation sentimentale, et qui se situe dans la tradition d'une littérature d'apprentissage, n'est plus chez Elfriede Jelinek que le porte-voix de ce qui reste après l'atomisation de ce qui est convenu d'appeler l'identité. Des morceaux de sens traversés et recollés entre eux par de mesquins espoirs, des désirs tordus, de dévorantes ambitions, des restes de ce qu'autrefois on nommait passion et qui constituait le sujet même du théâtre et de la littérature. 


\section{Elfriede Jelinek «lch will seicht sein»}

16 Ich will nicht spielen und auch nicht anderen dabei zuschauen. Ich will auch nicht andere dazu bringen zu spielen. Leute sollen nicht etwas sagen und so tun, als ob sie lebten. Ich möchte nicht sehen, wie sich in Schauspielergesichtern eine falsche Einheit spiegelt: die des Lebens. Ich will nicht das Kräftespiel dieses «gut gefetteten Muskels» (Roland Barthes) aus Sprache und Bewegung - den sogenannten «Ausdruck» eines gelernten Schauspielers sehen. Bewegung und Stimme möchte ich nicht zusammenpassen lassen. Beim Theater Heute wird etwas enthüllt, wie, sieht man nicht, denn es werden im Hintergrund die Bühnenfäden dafür gezogen. Die Maschine also ist verborgen, der Schauspieler wird mit Geräten umbaut, angestrahlt und geht umher. Spricht. Der Schauspieler ahmt sinnlos den Menschen nach, er differenziert im Ausdruck und zerrt eine andere Person dabei aus seinem Mund hervor, die ein Schicksal hat, welches ausgebreitet wird. Ich will keine fremden Leute vor den Zuschauern zum Leben erwecken. Ich weiß auch nicht, aber ich will keinen sakralen Geschmack von göttlichem zum Leben erwecken auf der Bühne haben. Ich will kein Theater. Vielleicht will ich einmal nur Tätigkeiten ausstellen, die man ausüben kann, um etwas darzustellen, aber ohne höheren Sinn. Die Schauspieler sollen sagen, was sonst kein Mensch sagt, denn es ist ja nicht Leben. Sie sollen Arbeit zeigen. Sie sollen sagen, was los ist, aber niemals soll von ihnen behauptet werden können, in ihnen gehe etwas ganz anderes vor, das man indirekt von ihrem Gesicht und ihrem Körper ablesen könne. Zivilisten sollen etwas auf einer Bühne sprechen!

17 Vielleicht eine Modeschau, bei der die Frauen in ihren Kleidern Sätze sprechen. Ich möchte seicht sein!

Modeschau deswegen, weil man die Kleider auch allein vorschicken könnte. Weg mit den Menschen, die eine systematische Beziehung zu einer ersonnenen Figur herstellen könnten! Wie die Kleidung, hören Sie, die besitzt ja auch keine eigene Form, sie muß um den Menschen gegossen werden, der ihre Form IST. Schlaff und vernachlässigt hängen die Hüllen, doch dann fährt einer in sie, der spricht wie mein Lieblings Heiliger, den es nur gibt, weils auch mich gibt: Ich und der, der ich sein soll, wir werden nicht mehr auftreten. Weder einzeln noch gemeinsam. Sehen Sie mich genau an! Sie werden mich nie wieder sehen! Bedauern Sie es! Bedauern Sie es jetzt. Heilig heilig heilig. Wer kann schon sagen, welche Figuren im Theater ein Sprechen vollziehen sollen? Ich lasse beliebig viele gegeneinander antreten, aber wer ist wer? Ich kenne diese Leute ja nicht! Jeder kann ein anderer sein und von einem Dritten dargestellt werden, der mit einem Vierten identisch ist, ohne daß es jemandem auffiele. Sagt ein Mann. Sagt die Frau. Kommt ein Pferd zum Zahnarzt und erzählt einen Witz. Ich will Sie nicht kennenlernen. Auf Wiedersehn.

Die Schauspieler haben die Tendenz, falsch zu sein, während ihre Zuschauer echt sind. Wir Zuseher sind nämlich nötig, die Schauspieler nicht. Daher können die Leute auf der Bühne vage bleiben, unscharf, Accessoires des Lebens, ohne die wir wieder hinausgingen, die Handtaschen in die schlaffen Armbeugen geklebt. Die Darsteller sind unnötig wie diese Tascheln, enthalten, gleich schmutzigen Taschentüchern, Bonbonsdosen, Zigarettenschachteln, die Dichtung! die in sie abgefüllt wurde. Verschwommene Gespenster! Produkte ohne Sinn, ist ihr Sinn doch das «Produkt einer überwachten Freiheit» (Barthes). Für jeden Spielzug auf der Bühne gibt es eine so oder so große Freiheitsmenge, von der sich der Schauspieler bedienen darf. Die Lacke 
Freiheit ist da, und der Schauspieler, nehmen Sie sich bitte!, holt sich seinen Saft, sein Kammerwasser, seine Sekrete. Daran ist nichts Geheimes. Er klebt seinen Rotz daneben. Aber was und wieviel er sich auch nimmt von seinem Teil an Gesten, Herumstolzieren, Plappern, muß imitiert werden können, denn er und andre wie er müssen es genauso nachmachen können. Wie Modebekleidung: Jedes Teil ist gleichzeitig definiert, aber nicht zu eng umgrenzt in dem, wozu es dienen soll. Der Pullover, das Kleid, auch sie haben ihre Spielräume und Armlöcher. Ja. Und was eben unbedingt nötig ist: wir! Wir haben nicht die Freiheit, falsch zu sein. Die auf der Bühne aber schon, denn sie sind Ornamente unsres Lebens, beweglich, abnehmbar von der Hand Gottes, des Regisseurs. Und dann reißt er einen ganzen Kragen Mensch herunter und leimt uns mit einem andren, der ihm besser gefällt. Oder das Sackkleid Mensch wird einfach kürzer gemacht, indem er den Saum neu absteckt, dieser Filialleiter von einer Spielzeugladenkette. Belästigen Sie uns nicht mit Ihrer Substanz! Oder womit immer Sie Substanz vorzutäuschen versuchen, wie Hunde, die sich mit aufgeregtem Getön umkreisen. Wer ist der Chef? Maßen Sie sich nichts an! Verschwinden Sie! Theater hat den Sinn, ohne Inhalt zu sein, aber die Macht der Spielleiter vorzuführen, die die Maschine in Gang halten. Nur mit seiner Bedeutung kann der Regisseur die leeren Einkaufstüten zum Leuchten bringen, diese schlappen undichten Sackein mit mehr oder weniger Dichtung drin. Und plötzlich bedeutet das Bedeutungslose was! Wenn der Herr Regisseur in die Ewigkeit hineingreift und etwas Zappelndes herausholt. Dann ermordet er alles, was war, und seine Inszenierung, die doch ihrerseits auf Wiederholung gegründet ist, wird zum Einzigen, das sein kann. Er verleugnet das Vergangene und zensiert gleichzeitig (Mode!) das Zukünftige, das sich nun für die nächsten Saisonen nach ihm zu richten haben wird. Das Zukünftige wird gezähmt, das Neue geregelt, bevor es noch eingetreten ist. Dann vergeht ein Jahr, und die Zeitungen schreien wieder vor Freude über ein Neues, Unberechenbares, das das Alte ablöst. Und das Theater beginnt wieder von vorn, die Vergangenheit kann von der Gegenwart abgelöst, erlöst werden, die sich aber, im ewigen Vergleich, über die Vergangenheit neigen muß. Dafür gibt es die Theater Zeitschrift. Man muß alles gesehen haben, um überhaupt etwas sehen zu können.

Doch nun zu unsren Mitarbeitern: Wie entfernen wir diese Schmutzflecken Schauspieler aus dem Theater, daß sie sich nicht mehr aus ihrer Frischhaltepackung über uns ergießen und uns erschüttern, ich meine überschütten können? Denn diese Leute sinds doch, die sich verkleiden und mit Attributen behängen, die sich ein Doppelleben anmaßen. Diese Personen lassen sich vervielfältigen, ohne daß sie ein Risiko eingingen, denn sie gehen nicht verloren. Ja, sie spielen nicht einmal mit ihrem Sein herum! Sie sind ja immer dasselbe, nie brechen sie durch den Boden oder erheben sich in die Luft. Sie bleiben belanglos. Schließen wir sie als Inventar aus unsrem Leben einfach aus! Klopfen wir sie platt zu Zelluloid! Wir machen vielleicht einen Film aus ihnen, von wo uns ihr Schweiß, Symbol einer Arbeit, der sie im Luxus ihrer Persönlichkeiten zu entkommen trachteten, nicht mehr anwehen kann. Aber ein Film als Theater, nicht ein Film als Film! Einfach draufhalten und abdrücken! Wie es liegt, so pickt es. Nichts kann mehr geändert werden und unterläuft damit die ewige Wiederholung des Nie Ganz Gleichen. Sie werden einfach aus unserem Leben verbannt und auf Lochstreifen gestanzt, die wacklige Melodien winseln. Fallen aus unserer Körperbetrachtung und werden zur Fläche, die vor uns abläuft. Werden unmöglich und müssen deshalb auch gar nicht erst verboten werden, denn sie sind nicht und nichts mehr. Oder auch: es werden bei jeder Vorstellung alle komplett ausgewechselt und 
machen jedes Mal etwas ganz Neues. Sie haben einen Vorrat an möglichen Spielzügen, aber nichts wird, ähnlich unserer Kleidung, ganz genauso wiederholt wie es war. Nur die Zeit bedroht uns alle mit dem Vergehn! Theater darf es nicht mehr geben. Entweder das Immergleiche wird immer gleich wiederholt (Filmabnahme einer geheimen Aufführung, die von uns Menschen nur mehr in ihrer EINZIGEN EWIGEN Wiederholung gesehen werden darf, oder keine zweimal dasselbe! Immer etwas ganz andres! Sowieso dauert nichts ewig, im Theater können wir uns drauf vorbereiten, in die Zeitlichkeit einzugehen. Die Bühnenmenschen treten nicht auf, weil sie etwas sind, sondern weil das Nebensächliche an ihnen zu ihrer eigentlichen Identität wird. Ihr Herumfuchteln, ihre plumpen, verwaschenen Aussagen, von Uneinsichtigen in ihre Mäuler gestopft, ihre Lügen, nur daran kann man sie voneinander unterscheiden. Ja, sie treten an die Stelle der Personen, die sie darstellen sollen und werden zum Ornament, zu Darstellern von Darstellern, in endloser Kette, und das Ornament wird auf der Bühne das Eigentliche. Und das Eigentliche wird, Platz! Zurück! zur Zierde, zum Effekt. Ohne sich um die Wirklichkeit zu kümmern, wird der Effekt zur Realität. Die Schauspieler bedeuten sich selbst und werden durch sich definiert. Und ich sage: Weg mit ihnen! Sie sind nicht echt. Echt sind nur wir! Wir sind das meiste, das es gibt, wenn wir schlank und schick in unsren eleganten Theaterkleidern hängen. Richten wir die Blicke nur noch auf uns! Wir sind unsere eigenen Darsteller. Brauchen wir nichts außer uns! Gehen wir in uns hinein und bleiben wir drinnen, jeder hofft ja, daß ihn möglichst viele betrachten mögen, wenn er durch die Welt stolziert, von den Zeitschriften und deren Bildern ordentlich geregelt wie eine gut geölte Maschine. Werden wir unsre eigenen Muster und sprenkeln wir den Schnee, die Wiesen, das Wissen, womit? Mit uns selbst! So ist es gut.

\section{« Sujet impossible » Gespräch mit Elfriede Jelinek}

Yasmin Hoffmann : In Österreich haben gerade Wahlen stattgefunden, in denen Sie eine Rolle gespielt haben, die Sie in dieser Form bestimmt nicht spielen wollten: Sie sind u.a. von den sogenannten "Freiheitlichen» (unter der Leitung von Jörg Haider) auserwählt und zum politischen Gegner erklärt worden; was konkret so aussah, daß in ganz Österreich folgende Wahlplakate zu sehen waren: «Lieben Sie Scholten, Jelinek, Häupl, Peymann, Pasterk... oder Kunst und Kultur? Freiheit der Kunst statt sozialistischer Staatskünstler. Die Wiener Freiheitlichen».

Persönlichkeiten aus dem politischen Leben - Rudolf Scholten, Kunstminister, Michael Häupl, Bürgermeister von Wien, Ursula Pasterk, Kultur stadträtin, - und zwei Persönlichkeiten aus der Theaterwelt werden von Jörg Haider angegriffen und als symbole für sozialistische Staatskünstler angeprangert. Wie erklären Sie sich, daß eine solche Rufmordkampagne in Österreich möglich ist? Welche Werte sind es, die Sie für die FPÖ vertreten? Diese Inszenierung erinnert doch ohne den geringsten Zweifel an «entartete Kunst».

Elfriede Jelinek : Das Infame an diesem Plakat ist eben, daß ich als einzige Künstlerin explizit angegriffen worden bin, denn auch Peymann wird ja nicht in seiner Eigenschaft als Regisseur, sondern als Theaterdirektor angegriffen. Indem man mich mit Politikern auf eine Stufe stellt, entwertet man meine Arbeit als simple plakative Politkunst, ohne ästhetischen Gehalt. Die F-Partei sagt ja: Als Künstlerin kann sie machen, was sie will, aber wo sie sich als Politikerin betätigt, wird sie von uns angegriffen. Es ist also gleichzeitig eine Anprangerung, daß ich entartete Kunst mache und gleichzeitig die 
Entwertung, daß ich eigentlich überhaupt keine Kunst mache, sondern Politik. Das Entscheidende daran ist, daß man mir (und auch Kollegen wie Roth, Turrini, etc.) vorwirft, Staatskunst zu machen, also in der Kunst die Geschäfte des Staates zu besorgen, diesem nach dem Mund zu reden, obwohl ich (und auch die genannten Autoren) mich immer als Kritikerin der Gesellschaft verstanden habe. Auch diese ästhetische Aufgabe, die ich mir gesetzt habe, wird hier desavouiert und dem Spott der Öffentlichkeit preisgegeben. Im wahrsten Sinne des Wortes will eine politische «Bewegung» (als solche versteht sich diese Partei wieder, das ist reine Nazidiktion!) hier eine Einzelperson, die nirgendwo eingebunden ist und nur sich selbst in ihrer Arbeit vertritt, eine Kritikerin der Gesellschaft mundtot machen, geradezu auslöschen. Wobei so gut wie keine Solidarität der Kollegen dazu erfolgt ist.

Y.H. : Bleiben wir bei dem politischen Engagement: in der Nora läßt sich noch ein individuell/ kollektives Sujet ausmachen, und ganz allgemein bleibt das Stück mit der Tradition eines politisch engagierten Theaters verbunden, in den folgenden Stücken scheint mir, daß Sie sich immer mehr von einem Subjekt-orientierten Theater entfernen...

E.J. : In der Nora gibt es dieses Subjekt noch, das ist richtig, es handeln noch wirkliche, voneinander deutlich abgegrenzte Personen, aber sie vertreten nicht eigentlich sich, sondern sie sprechen ihre Klasse, also: nicht sie sprechen, sondern ES spricht aus ihnen. Also ist das Subjekt auf der anderen Seite wiederum aufgehoben in dem Zusammenhang, daß individuelles Handeln wieder nicht möglich ist, sondern nur eine Illusion. Kraft der Komplexität und Geschlossenheit der Gesellschaft handelt jeder nicht wie er will, sondern wie er MUSS, der Spielraum für den einzelnen ist relativ klein, das Individuelle eine Illusion, das will ich zeigen.

Y.H. : Die meisten Ihrer Stücke sind historisch vorbelastet (Was geschah nachdem Nora ihren Mann verlassen hatte bezieht sich auf Ibsen, Clara. S. auf Clara Schumann und D'Annunzio, Krankheit oder Moderne Frauen u.a. auf die Geschwister Brontë, etc), die Figuren haben also schon eine historische oder literarische Vergangenheit, stammen aus einer Tradition, Emily sagt in der Krankheit: «Ich zum Beispiel komme aus einer langen Röhre hervor: der Vergangenheit». Was bedeutet die zu einer Röhre verkommene Vergangenheit?

E.J. : Die Röhre, die, wie ein Abflußrohr, die Menschen ausspuckt, ist : jeder ist so wie schon einmal ein anderer gewesen ist, obwohl er das (im Gegensatz zu Handkes Kaspar, in dem die Hauptfigur eben genau das sein will und als solcher dann wieder ein einzelner und unverwechselbar. Das will eben jeder, ich zeige aber, daß es eine Illusion ist, unverwechselbar sein zu wollen) eben nicht sein kann. Emily kommt, weil eine Emily Brontë (viele Emilys) einmal gewesen ist, aus dieser Röhre hervor, die vervielfältigt, viele enthält, die so gewesen sind wie sie. Wobei in diesem Fall ja nicht so viele Emilys gewesen sind, die meisten Frauen haben ja den bequemeren Weg der gesellschaftlich sanktionierten Mutterrolle gewählt.

Y.H. : Sie lassen in Kranheit oder Moderne Frauen einen Heiligen auftreten, den Sie auch in Ihrem Essay Ich will seicht sein zitieren und der folgende Rede hält: «Wer kann schon sagen, welche Figuren im Theater ein Sprechen vollziehen sollen? Ich lasse beliebig viele gegeneinander auftreten, aber wer ist wer? Ich kenne diese Leute ja nicht! Jeder kann ein anderer sein und von einem Dritten dargestellt werden, der mit einem Vierten identisch ist, ohne daß es jemandem auffiele». So austauschbar scheint mir die Verteilung der Aussagen in den bis jetzt genannten Stücken nicht, und ich kann mir nicht vorstellen, daß eben in einem Stück wie Kranheit z.B. die männlichen und weiblichen Rollen austauschbar sind. 
EJ. : Die Austauschbarkeit bezieht sich nicht auf die Figuren gegeneinander, die spielen, wie im Leben, «ihre Rollen», sondem jeweils auf ihre Gattung. So ist Helmer in der Nora eben Der Verwalter, Der Großbürger, und Nora ist Die Komplizin, sie handeln, wie soll ich sagen, typisiert, nicht individuell. Das wie der parodiert der Heilige gewissermaßen, indem er, wie ich das ja oft versuche, die Moral der Geschichte, die Wahrheit spricht, vergleichbar etwa den beiden Japanern am Ende der Raststätte. Die sogenannte licenza in der italienischen Barockoper. Das sind Figuren, die sagen, was der Autor mit dem ganzen will, und gleichzeitig parodieren sie die Figur der Autorin, stellen sie wieder in Frage. Der Heilige parodiert noch dazu das Theater, die Absurdität, daß Figuren gegeneinander antreten sollen, die nicht sie selbst sind, sondern einen anderen «spielen», in dem man aber wieder sich selbst erkennen soll, im Sinne der Mimesis und dann der Katharsis der griechischen Tragödie.

Y.H. : Die Gleichwertigkeit eines «anything goes» scheint mir auf Ihre ersten Prosa-Texte anwendbar (Michael, Lockvögel), aber wie sieht das in Ihrem Theater aus? Ist es nicht viel schwieriger im Theater die Vielfalt der Perspektiven einer sprechenden Person aufrecht zu halten als in der Prosa?

E.J. : Aus dem Gesagten geht schon, hoffe ich, hervor, daß ich eben die Vielfalt einer Person nicht als solche zeige, sondern die Einfalt in der Vielfalt, also ähnlich dem Brecht'schen Lehrstück: Daß das, was die Menschen am Ticken, am Laufen hält, eben relativ wenige Mechanismen sind, die sehr wohl beschreibbar sind, auch wenn jeder Mensch natürlich einzigartig und unverwechselbar ist, von seiner Anlage her (die Natalität Hannah Arendts, das hat sie ja gerade aus der Erfahrung des Faschismus hergeleitet). Diese Einzigartigkeit verrät aber die Gesellschaft dauernd, indem sie jeden von Anfang an manipuliert, vergesellschaftet. Ich versuche, eben um die Katharsis, also die Läuterung durch Erschrecken, herzustellen, eben diesen Entindividualisierungsprozeß, den die Gesellschaft dem Eineinen antut, in Sprache zu fassen, damit man erschrickt, wenn man das sieht. Überspitzt formuliert: Durch diesen Entindividualisierungsprozeß, den ich in meiner Theaterarbeit vorführe, versuche ich die Leute im Publikum zum Denken anzuregen, ihre Einzigartigkeit wiederzugewinnen, indem sie voll Schrecken erkennen, was aus ihnen gemacht worden ist.

Y.H. : In Ich will seicht sein behaupten Sie: «Ich will nicht spielen und auch nicht anderen dabei zuschaun. Ich will auch nicht andere dazu bringen zu spielen». Wie ist das zu verstehen? Sie schreiben doch für die Bühne?

E.J. : Natürlich will ich nicht ein Theater ohne Schauspieler, aber ich will Schauspieler, die sich und das, was sie tun, in ihrem Spiel dauernd selber auch hinterfragen, in Frage stellen. Mich interessiert die «interface» oder "the gap», der Abgrund, der Spalt zwischen dem Körper des Schauspielers und seiner Rolle, seinem Text (bei mir ist diese Rolle ja fast ausschließlich Sprache, und wenn sie nicht sprechen, sind sie nicht, meine Schauspieler). Da kann es ja zu ganz unterschiedlichen Ergebnissen kommen, und das ist, wenn überhaupt, das Faszinierende am Theater. Daß das Immergleiche, das Stück also, so unterschiedliche Möglichkeiten der Interpretation bietet, während der Film eben immer gleich abläuft. So ist am Theater nicht einmal eine Vorstellung desselben Stücks wie die andere: also ein Theorie der Wiederholung sollte dieser kleine Essay auch sein.

Y.H. : Eines der Merkmale Ihres Theaters ist die Negation und die Aufhebung. Die verschiedenen Aussagen sind kaum für längere Zeit gültig als die, die notwendig ist, um sie auszusprechen. Wie «ernst» steht es um folgende Aussage aus dem eben zitierten Essay: «Nur die Zeit bedroht uns 
alle mit dem Vergehn! Theater darf es nicht mehr geben. Entweder das Immergleiche wird immer immer gleich wiederholt [...], oder keine zweimal dasselbe! Immer etwas ganz anderes " Ersteres, wie Sie selbst sagen, ist eine Eigenschaft des Films, eine trotz der Bewegung statische Auffassung, die Wiederkehr des Immergleichen im "Zeitalter der technischen Reproduzierbarkeit», die zweite Möglichkeit erinnert mehr an Happening und Performance, und schließt die erste vollkommen aus.

EJ. : Wie ich schon sagte, der Film als immergleiche Wiederholung, das Theater als Nicht Gleiche Wiederholung. Und in Totenauberg habe ich beides bewußt zusammengebracht, indem ich auf der Bühne eine Leinwand aufbaue und die beiden Arten der Wiederholung gegeneinandersetze.

Y.H. : Immer noch in dem gleichen Text wird dem Zuschauer ein großer Platz eingeräumt. Brecht war ja der erste, der sehr konsequent den "Autor als Produzenten» sah und dem Zuschauer in dieser Produktion einen Platz zuteilt, der ihn dazu auffordert, sich künstlerisch, $d$. $h$ auch als dialektisches Subjekt zu verhalten. Könnte man ähnliches auch von Ihrem Theater behaupten, also daß Ihr Theater auch eine "Zuschauerkunst» impliziert.

E.J. : Es wird ja im Theater nicht ein Objekt für ein Subjekt, also eine Inszenierung für Zuschauer geschaffen, sondern umgekehrt schafft die Inszenierung, also das Objekt, auch das Subjekt, in Rückwirkung auf das Publikum. Ich will schon Erkenntnisprozesse in Gang setzen, und manchmal gelingt das auch, das beweisen die heftigen (zum Teil heftigen) Reaktionen auf mein Theater. Die Elfriede Gerstl hat einmal über mein Theater geschrieben : Wer vor mir erschrickt, erschrickt vor sich selber.

Y.H. : Sie sagen, daß «es» auch im psychoanalytischen Sinne aus Ihren Figuren he raus spricht, nur werden die Worte so gut wie nie von einem Gegenüber aufgefangen, es bleibt bei einem Aufeinanderprallen von Monologen, in Wolken. Heim, fehlt zum Beispiel ein wesentliches dramaturgisches Merkmal: der Dialog, auch ist der imperative Ton in vielen Ihrer Stücke vorherrschend.

E.J. : Wolken. Heim, ist ähnlich der Bildbeschreibung von Heiner Müller insofern eine Ausnahme, als der Text überhaupt nicht mehr aufgeteilt ist. Das muß die Aufführung selbst leisten, und die Ergebnisse sind dabei natürlich noch unterschiedlicher als bei den anderen Stücken. Man kann sich «Dem Deutschen» als solchem besser nähern, denke ich, indem man die philosophisehen, politischen, polemischen Texte, die es «fassen» (wie ein Wasserhahn das Wasser, oder eben die Röhre der Vergangenheit aus Krankheit, gegeneinanderstellt, und plötzlich versteht man die Gegenwart besser, aus dieser Vergangenheit heraus. Es hätte natürlich unendlich viele andere Texte gegeben, das Deutsche zu beschreiben, das war eben mein Textkörper, den ich ihm, aus der Position der Außenseiterin heraus (Frauen haben noch nie eine besondere Rolle in der deutschen Kultur gespielt), geliehen habe, denn aus dem großen Abstand sieht man besser.

Y H. : Zum Problem der Darstellbarkeit und der Entstellung des Subjekts: kann man bei ihren Figuren überhaupt noch von Fassade, Maske, oder Tiefe sprechen?

E.J. : Darstellbarkeit... da gibt es auch unendlich viele Möglichkeiten. Man kann es mit Masken spielen, also völlig entpersönlicht, und man kann eine ganz neue Geschichte hinter die Texte stellen, sozusagen die Figuren wie in einem chemischen Prozeß herausdestillieren aus den Sprachflächen. Das hat z. B. Peymann bei der Raststätte in Wien gemacht, indem er eine Geschichte wie eine Folie hinter meine Sprachflächen geschoben hat, was bei diesem Stück wahrscheinlich einfacher ist als bei den anderen. 
41 Y.H. : Zur Allmacht des Regisseurs, die Sie in «Ich will seicht sein» stark angreifen, und die sich im Fall der CastorfInszenierung in Hamburg sehr negativ auf die Raststätte ausgewirkt hat. Wie erklären Sie sich die feindliche Einstellung Castorfs dem Stück gegenüber? Aus politischen Gründen? Oder sind Ihre Texte eine zu große Herausforderung an die Bühne?

E.J. : Das weiß ich nicht, weshalb diese (auch wieder sehr produktive!) Feindseligkeit Castorfs gegen das Stück. Es gibt ja Künstler, die nur aus der Negativität heraus etwas entwickeln können, übrigens gehöre ich ja auch dazu. Vielleicht wäre diese Inszenierung als Uraufführung nicht gut gewesen, aber als eine von mehreren Inszenierungen ist sie ja sehr interessant, weil eben auch Aggressionen meinen Texten gegenüber interessant sind, das Publikum hat ja davon genügend, und meist richtet es sie gegen die Autorin, also diejenige, die versucht, die Wahrheit zu sagen, ihre Wahrheit, und nicht gegen die Verhältnisse, die zu kritisieren versucht wird. Es wird sozusagen stellvertretend der Bote geprügelt. Die ideale Inszenierung gibt es nicht. Es gibt nur eine, die mich mehr interessiert als die andere, und ich habe die Erfahrung gemacht, daß mich auch eine ganz gegen den Text gerichtete Inszenierung sehr interessieren kann, wenn auch ich eine neue Wahrheit über meine eigenen Texte darin erfahre.

Y.H. : Der Vampirismus zieht sich wie ein roter Faden durch Ihre Prosa und Ihr Theater, ohne daß man behaupten könnte, daß es sich dabei um eine einheitliche Metapher handelt. In Krankheit z. B. habe ich den Eindruck es handle sich um eine tragischkomische Alternative zur Männerwelt, wogegen in Texten wie Wolken. Heim. oder Ihrem letzten Roman Die Kinder der Toten der Vampirismus zu einer politisch historischen Metapher wird.

E.J. : Der Vampirismus ist so vielfältig, daß ich hier gar nicht alles dazu sagen kann, nur ein paar Anhaltspunkte. Einerseits steht er, wie in Wolken. Heim., für das Untote der deutschen Geschichte, die nie verschwindet, sondern wie die Hand dem Kind in dem deutschen Märchen immer wieder aus dem Grab herauswächst, bis die Mutter kommt und mit der Gerte draufschlägt. Die deutsche, die österreichische Geschichte kann nicht sterben, denn sie bringt immer wieder hervor, was sie schon einmal fast ausgelöscht hat. Andererseits steht er, wie in Krankheit, für die Frau schlechthin, also für ihre vampirische Existenz, da sie, wie Eva Meyer sagt, immer wieder auftaucht, während sie mit dem Verschwinden beschäftigt ist. Also die Werke der Frau sind vergängliche, hinfällige, andere werden ihr nicht zugestanden. Sie ist da und wieder nicht da, weil ihr nichts Dauerhaftes zugetraut und anvertraut wird, nur das Kind darf sie produzieren, und auch da könnte die männliche Technologie sie als Gebärerin, die einzige Rolle, die ihr gestattet wird, wieder abschaffen. Also sozusagen meine eigene Verzweiflung als «Schöpferin», die in ständiger Überschreitung etwas tut, was ihr nicht zugestanden wird, denn die großen Schöpfungen werden nur dem Mann zugetraut. In den Kindern der Toten ist es dann so, daß alles kulminiert im Unheimlichen. Dadurch, daß unsere Kultur so viele Opfer gefordert hat (im deutschen, im österreichischen Nazismus), sind wir alle zu lebenden Toten geworden, haben wir alle unser Leben sozusagen verwirkt. Die toten Lebenden und die lebenden Toten, das ist das Ergebnis.

Y.H. : In einem ganz anderen Sinne als bei Thomas Bernhard würde ich von Ihrem Theater ebenfalls behaupten, daß Sie das Übermaß, das Exzessive zur Kunst, zur «Übertreibungskunst» erhoben haben.

E.J. : Was Bernhard geschrieben hat, entpuppt sich ja, so wie die österreichische. Gesellschaft sich derzeit $\mathrm{zu}$ entwickeln scheint, leider überhaupt nicht als Übertreibung, sondern als absolut realistische Analyse. Seine Übertreibung liegt 
weniger im Inhaltlichen als im Sprachlichen, deshalb ist er ja ein so großer Stilist. In seinen Tiraden, die in die Atemlosigkeit hineinschwingen, wenn man versucht, dabei mitzuatmen, entsteht eigentlich im prosodischen Sinn (obwohl man Prosodie eigentlich nur auf die Lyrik anwendet) ein überlebensgroßer, atmender Text. Ich dagegen arbeite mehr im musikalischen Sinne mit dem Wort selbst, indem ich durch Alliterationen, Assonanzen, Metathese, etc. seine Wahrheit heraushole, aus dem Klang des einzelnen Wortes, es ist also ein kompositorisches Verfahren, daß ich die Sprache, auch gegen ihren Willen, zwingen möchte, nur durch sich selbst die Wahrheit preiszugeben. Wenn man will, eben ein kompositorisches Verfahren, während Bernhard eher mit dem Rhythmus arbeitet als mit dem einfachen Wort. doch wieder, auf andere Weise, hervortritt !

\section{NOTES}

1. Les dates renvoient aux dates de publication des originaux en Allemagne. Pour les titres français ils sont tous parus aux Éditions J. Chambon, Nîmes.

2. Éditions de l'Arche, Paris, 1994, traduction de J.-C. de Sirjac, mise en scène de Claudia Stavisky au Théâtre de la Colline en janvier 1994.

3. Ed. J. Chambon, Nîmes 1994, une lecture de cette pièce fut proposée dans le cadre de la semaine du théâtre autrichien au Centre Pompidou, en décembre 1995.

4. Heiner Müller zitiert in Friedrichs, Regine : «Nachwort zu Krankheit oder Moderne Frauen», Prometh-Verlag, Köln, 1987, p. 98.

5. Henrichs, Benjamin: «Biß zum Tode. Theater in wilder Zeit», in Die Zeit, Hambourg, le 27.02.1987, p. 60 .

6. In Jelinek, Theaterstücke, Prometh Verlag, Köln, 1984.

7. Prometh-Verlag, Köln, 1987.

8. Artaud, Antonin, Le Théâtre et son double, NRF, Gallimard, Paris, 1964, p. 42.

9. Bachmann, Ingeborg : «Vorrede zum Fall Franza », in Werke, t. 3, PiperVerlag, München 1982, p. 342.

10. «Sur la brèche, sans fard, avec artifices : Entrevue avec Elfriede Jelinek » réalisée par HansJürgen Greif, in Nuit Blanche, n54, déc. 1993, Québec, Canada, p. 38. 


\section{RÉSUMÉS}

«Sujet impossible » n'est pas qu'une boutade. Le théâtre d'Elfriede Jelinek étant difficile à classer dans un genre particulier, tant il transgresse les règles du jeu dramatique, "sujet impossible " s'applique autant à l'impossibilité du sujet à se constituer qu'aux sujets impossibles auxquels Elfriede Jelinek s'attaque. Sujet conçu en trois volets, pour laisser le plus de place possible à l'auteur: une brève introduction à son œuvre théâtrale à travers deux pièces représentatives : Krankheit oder Moderne Frauen et Was geschah, nachdem Nora ihren Mann verlassen hatte, suivi d'un essai d'Elfriede Jelinek : «Ich will seicht sein» et d'un entretien centré sur les problèmes liés à la constitution du sujet théâtral.

"Sujet impossible» ist nicht nur ein Wortspiel. Gemeint sind die unmöglichen Themen, die Elfriede Jelinek auf die Bühne bringt, aber auch die Unfähigkeit einer Subjektkonstituierung. Diese unmöglichen Stücke in eine bestimmte Gattung einzuordnen, ist nicht einfach, da sie ständig die Grenzen des Vorstellbaren oder Darstellbaren überschreiten. Daher der Versuch, ihr Theater unter drei Aspekten darzustellen: einer kurzen Einführung anhand von zwei repräsentativen Stücken (Krankheit oder Moderne Frauen, Was geschah, nachdem Nora ihren Mann verlassen hatte), einem Essay von Elfriede Jelinek («Ich will seicht sein») und einem Gespräch mit der Autorin, in dem hauptsächlich die Probleme der Subjektkonstituierung auf der Bühne angesprochen werden.

\section{AUTEUR}

\section{YASMIN HOFFMANN}

Université d'Orléans 